\title{
Development of Integrated Portable Device and Mobile Apps for Homecare System: Body Temperature and Respiration Rate
}

\author{
Edgina Honesta, Agung W. Setiawan \\ Institut Teknologi Bandung, Jl. Ganesa No. 10, Bandung 40132, Indonesia
}

\begin{tabular}{l}
\hline ARTICLE INFO \\
\hline Article history: \\
Received April 02, 2021 \\
Revised April 17, 2021 \\
Accepted April 21, 2021 \\
\hline
\end{tabular}

\section{Keywords:}

Android-based;

Body temperature;

Portable;

Respiratory rate

\begin{abstract}
A Homecare system is a system that collects patient' vital signs and health information. Vital signs abnormalities appear several hours before the patient health quality decrease. Therefore, the home care system can be applied to prevent chronic diseases. It is estimated that $25 \%$ of chronic disease patients can be prevented by checking vital signs regularly. In this research, an Android-based portable product development that integrates measurements of body temperature and respiration rate is developed. Body temperature will be detected by an infrared temperature sensor. A thermistor will be used to calculate the respiration rate. A thermistor is a resistor whose resistance is dependent on temperature. The accuracy achieved by body temperature measurement is $84 \%$ with $\pm 1.37 \%$ precision. The accuracy achieved by the respiration rate measurement is $98 \%$ with $\pm 3.98 \%$ precision. The two gauges are integrated into the microcomputer with a serial communication channel. And then, the measurement results that have been processed on the microcomputer are sent to Android using Bluetooth. Measurement results can be displayed and saved on the Android application. Product portability parameters are measured by weight, size, durability, and power supply. The power supply for this system uses a power bank where the measuring system can be turned on for 3 hours. This research can be applied to a homecare system that collects patients' vital signs and health information.
\end{abstract}

This work is licensed under a Creative Commons Attribution-Share Alike 4.0

Agung W. Setiawan,

Institut Teknologi Bandung, Jl. Ganesa No. 10, Bandung 40132, Indonesia

Email: awsetiawan@stei.itb.ac.id

\section{INTRODUCTION}

Homecare system is an application of telehealth that brings health care technology to patients in their homes. The home care system is designed to meet the needs of a range of patients, from those who require minimal monitoring to those who require sophisticated monitoring. The homecare system collects patients' vital signs and health information. Vital signs are very important to determine patients' health status. Several parameters need to be checked regularly, i.e., Blood pressure, body temperature, pulse rate, oxygen saturation, and respiration rate. These parameters can be used as the first indication of patients' physiological abnormality [1][2][3].

The homecare system can be applied to prevent chronic diseases. According to the World Health Organization (WHO), in 2020, the mortality rate that is caused by chronic diseases will increase to $73 \%$ of all causes. The most frequent and dominant chronic diseases are cardiovascular disease, cancer, chronic lung disease, and type 2 diabetes. It is important to check the vital signs regularly to prevent chronic diseases [4]. Homecare systems can increase patients' disease-specific knowledge, trigger earlier clinical assessment and treatment, improved self-management and shared decision-making [5]. Remote monitoring has been associated with reduced hospital admissions for people with chronic disease [6]. A homecare system can significantly decrease the need for hospital care for elderly patients whose disease towards chronic diseases without increasing total healthcare costs [7]. It is estimated that $25 \%$ of chronic disease patients can have benefited if their vital signs can be measured and monitored at home. Another $50 \%$ will have benefit from the integration of existing medical equipment into smartphones or other devices [8]. 
One of the developed products that integrate the measurement of vital signs is MouthLab. MouthLab is used for Rapid Medical Assessment (RMA), so the packaging is designed portable. MouthLab able to measure pulse rate, respiratory rate, body temperature, blood oxygen level, and blood pressure with only one device. The measurement of vital signs is done by the patient holding the instrument and placing their fingers in a certain position. Besides that, the mouthpiece must be set in the mouth. The measurement results of this tool are then sent via Bluetooth to a computer [9].

In other research, there is an Android-based product that can measure vital signs. This product integrates several sensors to measure body temperature, blood oxygen level, electrocardiogram (ECG), pulse rate, and respiratory rate. The result will be saved on the cloud-based database before being sent back to Android. The application needs to be used online. The doctor can also access the measurement result through his computer. This tool is applied to patients whose vital signs must be monitored by medical personnel continuously [10].

Currently, portable vital signs measurement products that can be used by patients and accesses directly through Android are being developed. Thus, this research integrates several vital signs measurements with the Android-based smartphone. The sensors and algorithms are chosen based on the best test results from several algorithms which we have obtained. The test results show that the measurement results have entered the FDA standard and can be saved directly to the smartphone so it can be used offline. This paper focuses on body temperature and respiration rate first. Because body temperature is one of the vital signs that most people pay attention to [11], and the respiration rate is one of the most ignored in clinical practice [12].

\section{PROPOSED SYSTEM}

\subsection{System Specifications}

In this study, two sensors are used to measure the body temperature and respiration rate. The body temperature will be acquired using an infrared temperature sensor. A thermistor will be used to measure the respiration rate. These sensors will be connected to a microcomputer, Raspberry Pi3. The infrared sensor input is the temperature of the forehead. For the respiration rate measurement, a thermistor is used to measure the temperature changes in the area around the nose and mouth that happened during respiration. The output of body temperature measurement is digital-based. Therefore, it will measure the decimal value of body temperature in Celsius. On the other hand, the output of respiration rate measurement is an analog signal which will be changed to digital-based using Analog-to-Digital Converter (ADC). The result of both measurements will be sent to an Android-based smartphone. There will be an application that is used to display and save the measurement results. The system diagram block of this research is shown in Fig. 1.

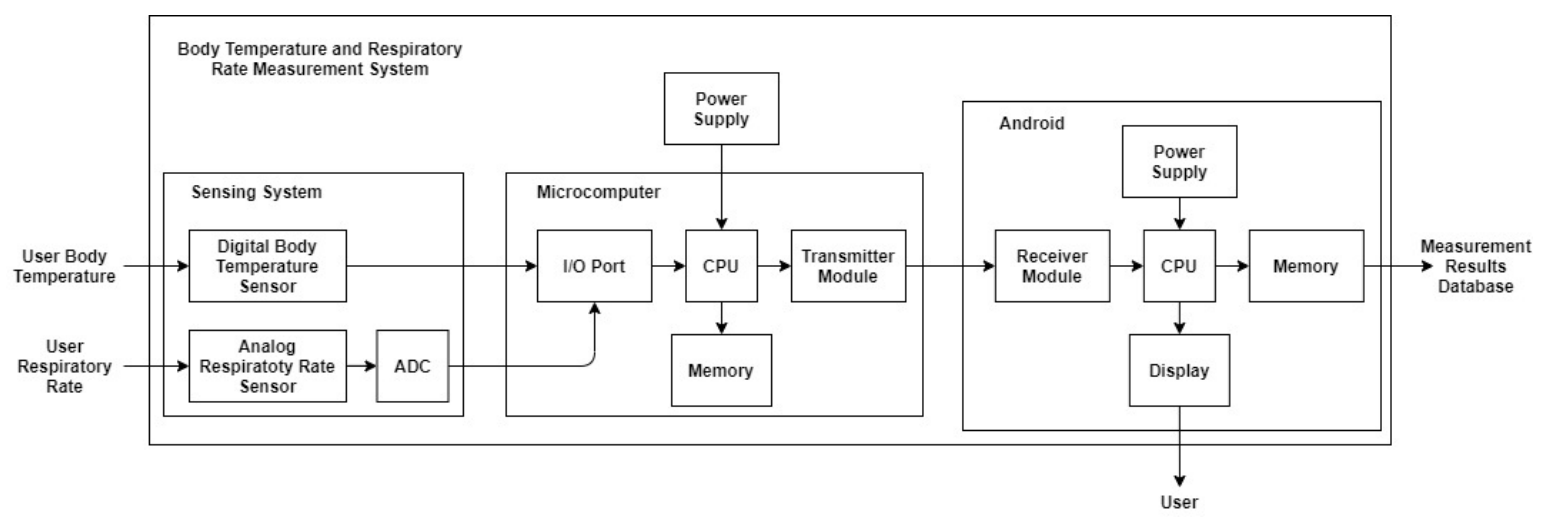

Fig. 1. System Diagram Block

The body temperature measurement is strongly influenced by the patients' age [11]. Elderly patients have temperatures lower than the adults' temperature with a difference of $0.23^{\circ} \mathrm{C}$. Normal body temperature is measured on the skin, which can be either axillary or forehead is in the range of $35.01^{\circ} \mathrm{C}-36.93^{\circ} \mathrm{C}$. The respiration rate of healthy adults at rest is around 12-18 breaths per minute [13]. The average respiration rate at rest according to age is as follows:

- $\quad$ born for up to 6 weeks: 30-40 breaths per minute;

- 6 months: $25-40$ breaths per minute;

- 3 years: $20-30$ breaths per minute;

- 6 years: $18-25$ breaths per minute;

- 10 years: $17-23$ breaths per minute [14];

- $\quad$ Adults: $12-18$ breaths per minute [13]; 
- Elderly $\geq 65$ years: breathing $12-28$ per minute;

- Elderly $\geq 80$ years: $10-30$ breaths per minute [15].

In this paper, there are three considerations taken while designing and implementing this system, which are (1) accuracy, (2) System Portability, and (3) Android Application - The Access and Storage of the Measurement Results.

According to the accuracy specification in [9], the accuracy standard for body temperature is $\pm 0.3^{\circ} \mathrm{C}$ and for respiration rate is \pm 3 BPM. The Android-based application can be easily used by users. Application usage satisfaction is assessed through the overall application, application appearance, ease of understanding the application, and usage in subjects of varying ages. System portability is assessed by measuring the weight, dimensions, durability, and power reliability [15]. Portable devices for the medical environment must be supplied with sufficient power that is capable of monitoring physiological parameters, displaying them for both doctors and patients, and storing the results for review.

There are two limitations in realizing the proposed system. First, the body temperature measuring tests are performed on healthy subjects, while respiration rate testing tests are carried out on healthy subjects with measurements in the normal and abnormal ranges. The second limitation is the type of smartphones that are supported is Android.

\subsection{System Realization}

The measurement of body temperature and respiration rate are done serially, as shown in Fig. 2. The I2C communication protocol is used on body temperature measurement, while the SPI communication protocol is used on the respiration rate measurement. Body temperature is first measured. The results are stored temporarily while waiting for the measurement of the respiration rate to finish. The microcomputer will process the results before sending them using Bluetooth. The microcomputer and Android must be paired first. Results are only sent to one Android that has been connected. An infrared sensor is used to measure body temperature. MLX90614 consists of several types. MLX90614 ESF DAA is the type of infrared sensor that is used. By using this type, the accuracy of measurement is according to medical accuracy, which is $0.2^{\circ} \mathrm{C}$. The required power supply is $3 \mathrm{~V}$. This sensor consists of 4 pins with two cables that are used as the communication line as the communication protocol used to retrieve data is I2C. In the infrared sensors circuit, pull-up resistors can affect the performance of the sensor. The calculation of pull-up resistor resistance is based on an equation, as in:

$$
R_{p(\min )}=\frac{V_{c C}-V_{O L(\max )}}{I_{O L}}
$$

The range of pull-up resistors that can be used based on the above calculation is $1.525 \mathrm{k} \Omega$ to $236 \mathrm{k} \Omega$. The consideration for determining the pull-up resistor used is to use a resistor that is at least ten times smaller than the input pin impedance value. As $R_{p(\max )}$ is $236 \mathrm{k} \Omega$, the impedance is ten times less than $R_{p(\max )}$ is $23 \mathrm{k} \Omega$. Based on the resistor are available, and according to the limit of the pull-up resistor, the selected pull-up resistor is ten $\mathrm{k} \Omega$. As the infrared sensor is affected by the measurement distance and its surroundings, a funnel is made with a height of $2 \mathrm{~cm}$ and a diameter of $1 \mathrm{~cm}$.

Respiration rate measurements were performed using a 10K NTC sensor. The measurement temperature range of this sensor is $-55^{\circ} \mathrm{C}$ to $125^{\circ} \mathrm{C}$. The NTC resistor is a resistor with a negative temperature coefficient, which means that as the temperature increases, the resistance will decrease. As the sensor output is an analog signal, therefore analog-to-digital converter is needed. MCP3008 is used as the analog-to-digital converter. The communication protocol of this ADC is SPI. Through MCP308, the microcomputer can retrieve the data.

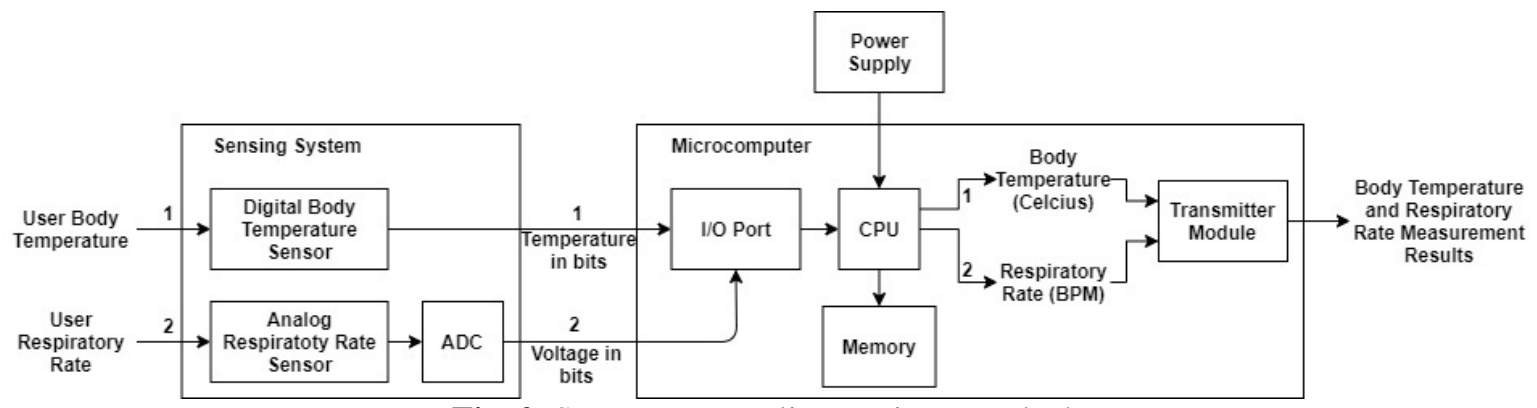

Fig. 2. System Data Delivery Diagram Block 
Respiration rate is measured by evaluating the temperature difference that occurred when breathing. One respiration cycle consists of one inhale, and one exhale. When the breath is exhaled, temperature around the nose and mouth will increase and vice versa. Thus, one respiration cycle can be counted when the resistance of the sensor rises and then falls. To have an accurate result, an appropriate algorithm to calculate the respiration rate is needed.

The implemented algorithm will calculate the respiration rate based on the peaks and valleys of the signal to calculate the respiration rate. The algorithm will receive input of measurement in decimal. The first 30 will be used as a lag to calculate the mean and standard deviation of the data. Based on the standard deviation, a coefficient times standard deviation is the data threshold to detect the peak and valley in the data. If the signal value minus the mean value is greater than the standard deviation that has been multiplied by the specified coefficient will be output as one or minus one. If the data is above the threshold, the output will be converted as one, and minus one if the data is below the threshold. If the signal value reduced by the mean value is not greater than the standard deviation multiplied by the coefficient, then the output is zero. Based on the output array, if the output moves from minus one to zero, the count will be incremented. Measurement is set to be done by 62 seconds.

As respiration rate measurement is done by measuring the temperature around the nose and mouth, a funnel is needed to isolate the environment. There are two alternatives that we have tried: testing it by using only a funnel and testing it by putting on a mask. Based on the test results, the alternative by using the funnel with a mask is better. Thus, the packaging is made with a funnel that has the same diameter as the mask nebulizer, so the funnel can be inserted into the mask nebulizer. The packaging for the respiration rate sensor that has been designed and made is as follows. The system will be put inside a suitcase. While designing the suitcase, it is expected to require a size of $\pm 30 \times 20 \times 10 \mathrm{~cm}^{3}$ with a mass of $\pm 2 \mathrm{~kg}$. Inside the suitcase, there is black foam to prevent shock from damaging the sensor. At the time of implementation, the aluminum suitcase purchased has a size of $27 \times 23 \times 7 \mathrm{~cm}^{3}$.

The application was made using the Android operation system. The application consists of five interfaces, as shown in Fig. 3. The first interface is for users or the examiner to fill in their information. The data will be saved in a database. The next interface is for filling in the patient information. The data will also be saved in a database. The third interface displays the steps to install the measuring tools. When the device is ready to be used, the user pressed the start button. The application will check the Bluetooth connection and send a command to the microcomputer to start the measurement. When the measurement is done, the application will display the result through the interface. The results will also be saved to the database. The application also has an interface that displays the measurement results, which contain all data such as the identity of the examiner, patient, and the measurement results.

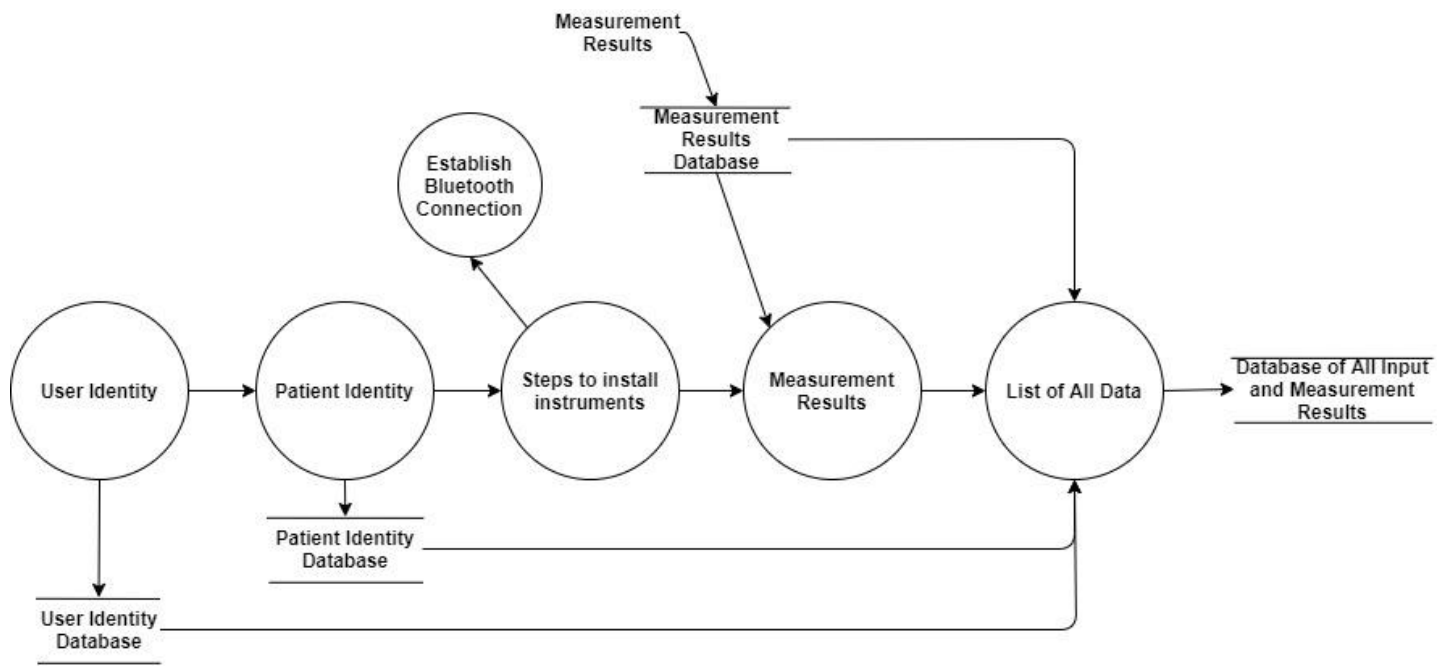

Fig. 3. User Interface Flow Diagram

\section{RESULTS AND DISCUSSION}

\subsection{Body Temperature and Respiration Rate Measurement Accuracy}

The accuracy and precision of body temperature measurement are tested by comparing the results of the measurement with the results of the digital thermometer measured through the axilla. The digital thermometer has an accuracy of $\pm 0.2^{\circ} \mathrm{C}$ with measurement time \pm 2 minutes. Tests were carried out on 45 healthy subjects. 
The time needed to measure body temperature is 60 seconds as the measurement using the MLX90614 sensor began to stabilize at 59 to 60 seconds, as shown in Fig. 4, which shows one of the measurement samples. However, the data still experienced a deviation of $\pm 0.02^{\circ} \mathrm{C}$ up to 70 seconds after the start of measurement. Therefore, the time needed for body temperature measurement is 60 seconds. Based on the FDA accuracy standard of $\pm 0.3^{\circ} \mathrm{C}$, the accuracy of the tested sensor is $84.44 \%$ with $\pm 1.37 \%$ precision and a $1 \%$ average error.

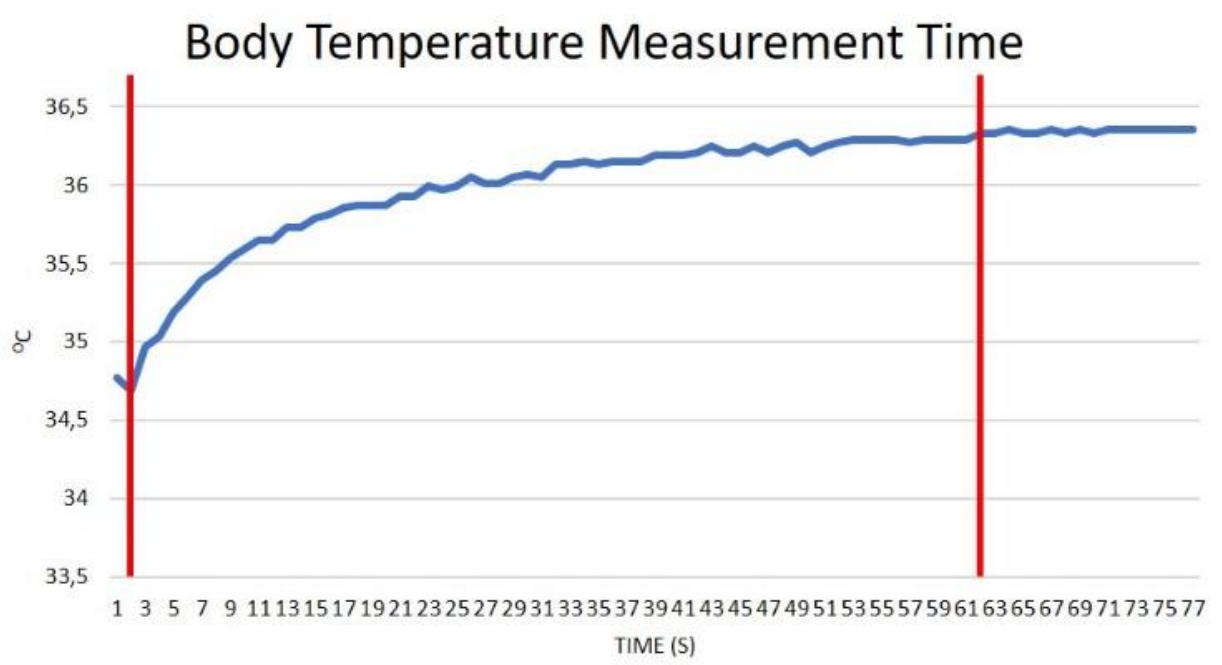

Fig. 4. One of The Measurement Samples Graph

Respiration rate testing is done by comparing the results of device measurement with the subject count. Tests were also carried out on 45 subjects. Based on the implemented algorithm, the respiration rate count is to calculate signal valleys that fall below the threshold. In Fig. 5, respiration rate is measured: 16 BPM, the cyan lines are the mean of the data, while green lines are the threshold. If the data passed over or falls below the threshold, the algorithm will output 1 or -1 . The respiration count is calculated by the count valley in the output. In Fig 1, respiration rate measured: 16 BPM, it is shown that the specified boundary base is correct. The subject count is 15 BPM, while the measurement result is 16 BPM. In Fig. 6, respiration rate is measured: $21 \mathrm{BPM}$, the subject count is $21 \mathrm{BPM}$, and the measurement result is $21 \mathrm{BPM}$.

Based on the specified specifications, if the deviation is within $\pm 3 \mathrm{BPM}$, then the measurement results will be consider measured accurately. The respiration rate measurement testing produces a result with an accuracy of $97.78 \%$, with $\pm 3.98 \%$ precision and 1.4 standard deviations.
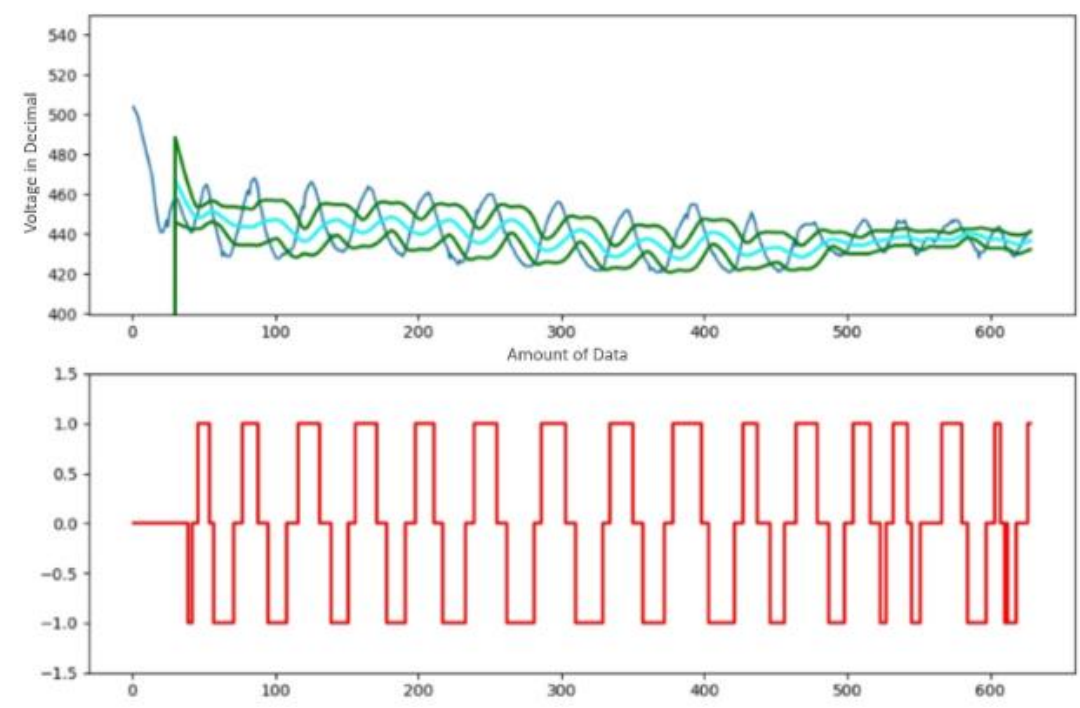

Fig. 5. Respiration rate measured: $16 \mathrm{BPM}$ 

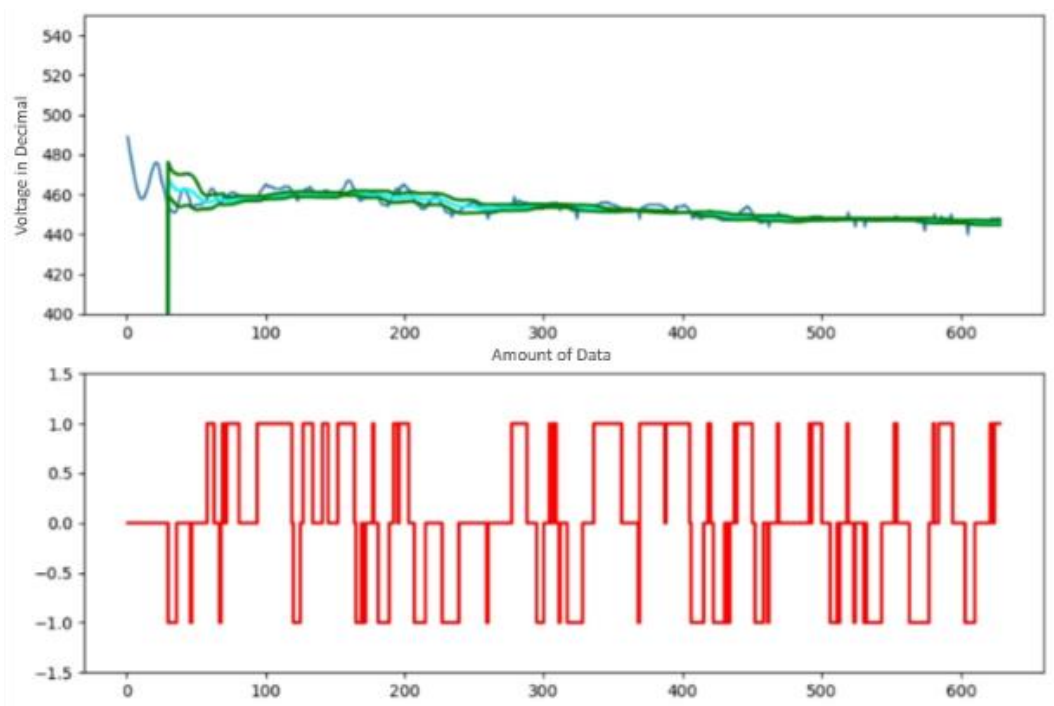

Fig. 6. Respiration rate measured: $21 \mathrm{BPM}$

\subsection{System Portability}

Product portability parameters are measured by weight, size, durability, and power supply. The product is loaded in a $27 \times 23 \times 7 \mathrm{~cm}^{3}$ suitcase with a weight of $1.06 \mathrm{~kg}$ that has good durability. The product is shown Fig. 7. Product resistance is checked by dropping the suitcase, then reused the device to measure body temperature and respiration rate. When the product is tested after dropping, the device still runs properly. Each time the measurement is estimated to require a power supply of $630 \mathrm{~mA}$. The power supply used in this test is a power bank with a current of $2.1 \mathrm{~A}$ with a capacity of $12000 \mathrm{mAh}$. The power supply is tested by operating the device until the power supply runs out. When tested, the measuring instrument can be operated for \pm 3 hours ( \pm 12 times the measurement) so as expected.

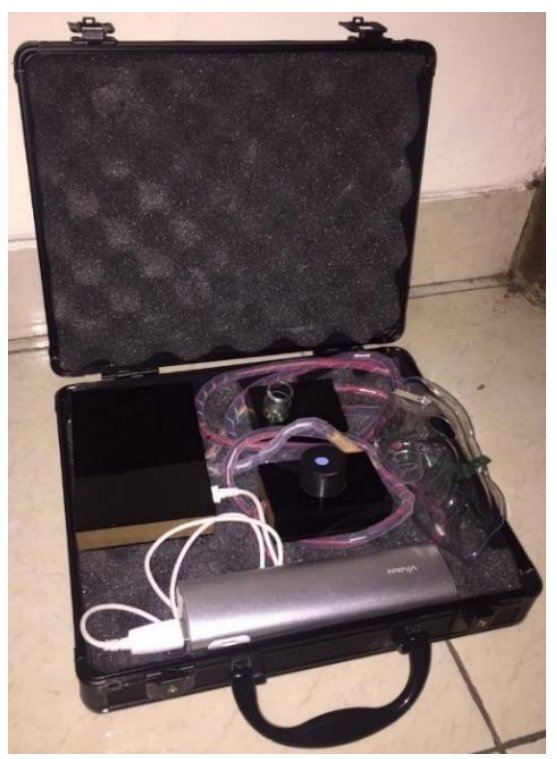

Fig. 7. Product in Suitcase

\subsection{Android Application - Measurement Results Access and Storage}

The application is made in Indonesia since the user that we tested are Indonesian. The application user interface is tested by explaining the purpose of the application and let the subject used it directly. Then, the subject will fill the prepared questionnaire. The application was tested on the whole application usability, application interface, ease of understanding the application, and the usage on subjects of varying ages. The test was done on 43 subjects of different ages. $49 \%$ of subjects aged 18-25 years, 30\% aged 25-34 years, $16 \%$ aged 35-49 years, and the rest aged over 50 years with education dominated by high school and bachelors. All 
subjects stated that the composition of the writing, the contents, and the button on display is clear and easy to understand. The flow of the application interface can also be understood by all subjects. For the ease of usage of the application, $58.1 \%$ stated that the application is very easy to use, while $30.2 \%$ stated it was quite easy, and the rest stated it not difficult to use and not easy. The application display how the users can use the products, as shown in Fig. 8. The measurement results were tested by displaying the results as shown in Fig. 9 and Fig. 10 that have been saved in the database.

The database can be accessed in a separate interface consisting of the entire input and measurement results. The database can also be exported in CSV format, as shown in Fig. 11 and Fig. 12. The testing is done by re-displaying the measurement data in the list interface and viewing the file that has been exported.

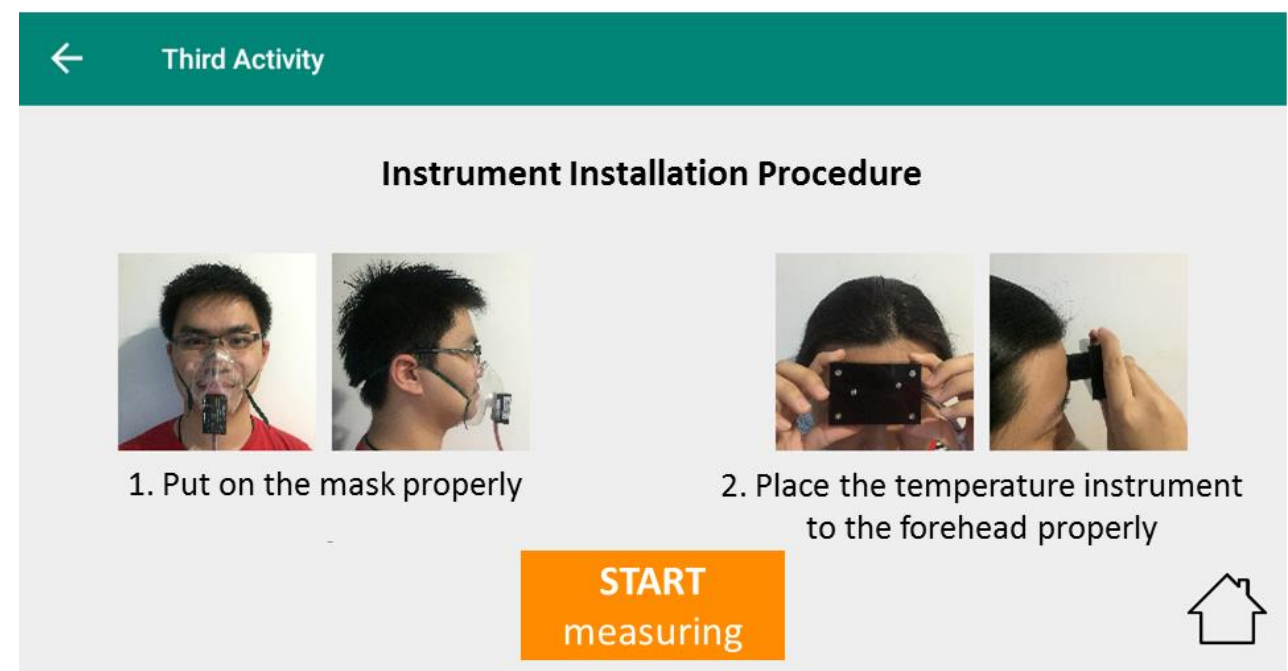

Fig. 8. How to use the product

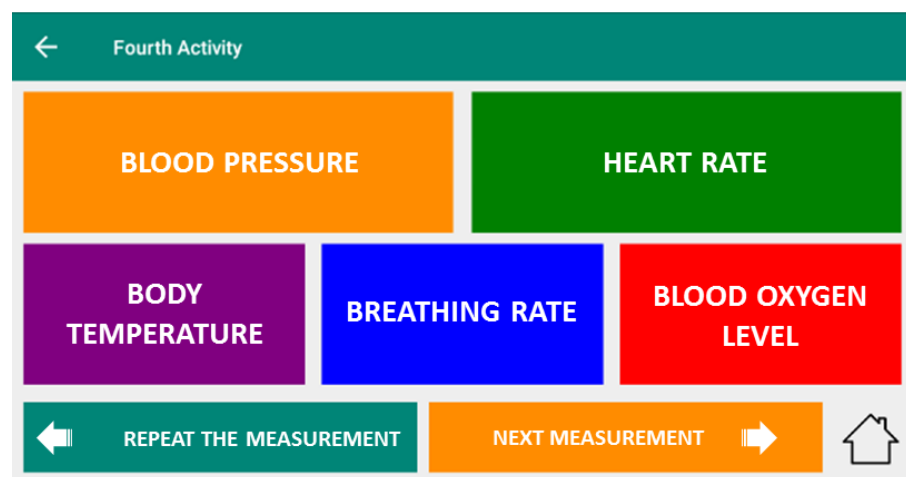

Fig. 9. Product Measuring Vital Signs

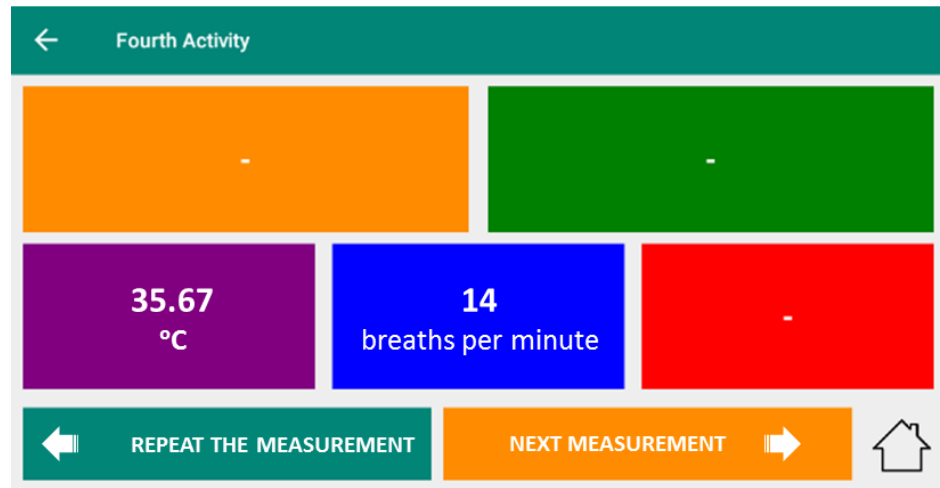

Fig. 10. Measuring Results 


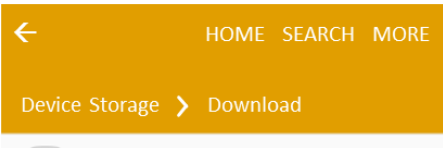

data.csv

Fig. 11. File .csv

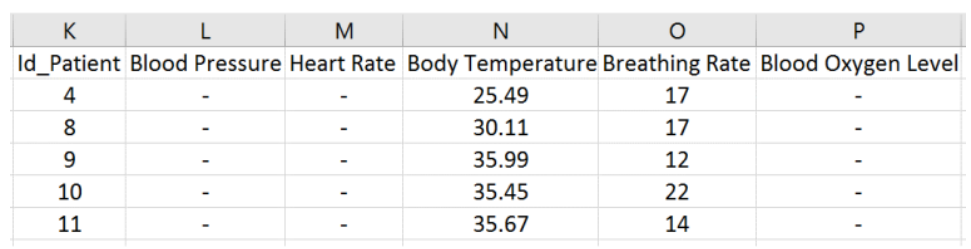

Fig. 12. Results in .csv

\section{CONCLUSION}

Android-based integration of measurement system of body temperature and respiration rate was successfully implemented. At this stage, the system has not been tested on unhealthy subjects. Body temperature measurement is performed using the MLX90614 infrared sensor reach accuracy of $84.44 \%$ with $\pm 1,37 \%$ precision. Respiration rate measurement is performed using the NTC sensor, which reaches an accuracy of $97.78 \%$ with $\pm 3.98 \%$ precision. The parameters of a portable product are products with a certain weight, dimensions, durability, and power supply. This product weighs $1.06 \mathrm{~kg}$ with $27 \times 23 \times 7 \mathrm{~cm}^{3}$ dimension and can last for 3 hours. The results of testing the application interface state that the majority feel that this application is very easy to use. Measurement data can be exported into CSV format. Besides, other vital signs measurements can be added, such as blood pressure, pulse rates, and blood oxygen levels. Based on the input from user interface testing, the color on the display that shows the measurement results should be replaced with a lighter color and added information of which results of vital sign measurement. For further developments, data storage can also be added to a cloud database so that it can be used offline and online also can be accessed by medical personnel associated with the users. It might be possible to make products that are more portable as in research [9]. This research can be applied to a homecare system that collects patients' vital signs and health information.

\section{REFERENCES}

[1] W. Q. Mok, W. Wang, and S. Y. Liaw, "Vital signs monitoring to detect patient deterioration: An integrative literature review," International Journal of Nursing Practice, vol. 21, no. S2, pp. 91-98, May 2015. https://doi.org/10.1111/ijn.12329

[2] G. Sun et al., "Vital-SCOPE: Design and Evaluation of a Smart Vital Sign Monitor for Simultaneous Measurement of Pulse Rate, Respiratory Rate, and Body Temperature for Patient Monitoring," Journal of Sensors, vol. 2018, 2018. https://doi.org/10.1155/2018/4371872

[3] J. Chaglla E., N. Celik, and W. Balachandran, "Measurement of Core Body Temperature Using Graphene-Inked Infrared Thermopile Sensor," Sensors, vol. 18, no. 10, pp. 3315, Oct. 2018. https://doi.org/10.3390/s18103315

[4] "WHO | Integrated chronic disease prevention and control," WHO, 2010, Accessed: Apr. 17, 2021. [Online].

[5] R. C. Walker, A. Tong, K. Howard, and S. C. Palmer, "Patient expectations and experiences of remote monitoring for chronic diseases: Systematic review and thematic synthesis of qualitative studies," International Journal of Medical Informatics, vol. 124, pp. 78-85, Apr. 2019. https://doi.org/10.1016/j.ijmedinf.2019.01.013

[6] J. Polisena et al., "Home telehealth for chronic obstructive pulmonary disease: A systematic review and metaanalysis," Journal of Telemedicine and Telecare, vol. 16, no. 3, pp. 120-127, Apr. 2010. https://doi.org/10.1258/jtt.2009.090812

[7] J. Lyth, L. Lind, H. L. Persson, and A. B. Wiréhn, "Can a telemonitoring system lead to decreased hospitalization in elderly patients?," Journal of Telemedicine and Telecare, vol. 27, no. 1, pp. 46-53, Jan. 2021. https://doi.org/10.1177/1357633X19858178

[8] "Monitoring and analysis of vital signs of a patient through a multi-agent application system." https://gredos.usal.es/handle/10366/130106 (accessed Apr. 17, 2021).

[9] G. Y. Fridman, H. Tang, D. Feller-Kopman, and Y. Hong, "MouthLab: A Tricorder Concept Optimized for Rapid Medical Assessment," Annals of Biomedical Engineering, vol. 43, no. 9, pp. 2175-2184, Sep. 2015. https://doi.org/10.1007/s10439-015-1247-1

[10] R. Abi Zeid Daou, E. Aad, F. Nakhle, A. Hayek, and J. Börcsök, "Patient vital signs monitoring via android application," in 2015 International Conference on Advances in Biomedical Engineering, ICABME 2015, Nov. 2015, pp. 166-169. https://doi.org/10.1109/ICABME.2015.7323278

[11] M. Sund-Levander, C. Forsberg, and L. K. Wahren, "Normal oral, rectal, tympanic and axillary body temperature in adult men and women: a systematic literature review," Scandinavian Journal of Caring Sciences, vol. 16, no. 2, pp. 122-128, Jun. 2002. https://doi.org/10.1046/j.1471-6712.2002.00069.x

[12] M. Elliott, "International Archives of Nursing and Health Care Why is Respiratory Rate the Neglected Vital Sign? A Narrative Review," 2016. https://doi.org/10.23937/2469-5823/1510050

[13] G. Yuan, N.A. Drost, and R.A. McIvor, "Respiratory rate and breathing pattern,” McMaster University Medical Journal, vol. 10, no. 1, pp. 23-28, 2013. 
[14] W.Q. Lindh, M. Pooler, C.D. Tamparo, B.M. Dahl, and J. Morris, "Delmar's comprehensive medical assisting: administrative and clinical competencies," Cengage Learning, April 2013.

[15] A. Rodríguez-Molinero, L. Narvaiza, J. Ruiz, and C. Gálvez-Barrón, "Normal Respiratory Rate and Peripheral Blood Oxygen Saturation in the Elderly Population," Journal of the American Geriatrics Society, vol. 61, no. 12, pp. 22382240, Dec. 2013. https://doi.org/10.1111/jgs.12580

\section{BIOGRAPHY OF AUTHORS}

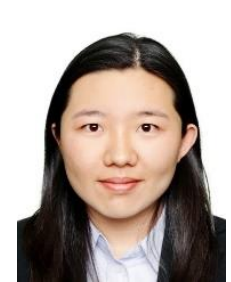

\section{Edgina Honesta}

She receives the bachelor's degree in biomedical engineering from the Institut Teknologi Bandung, Bandung, Indonesia, in 2019, respectively. She has an interest related to the Internet of Things (IoT) applications. Email: edginahonesta@gmail.com

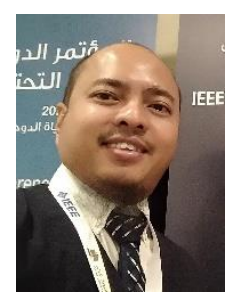

\section{Agung W. Setiawan}

He was born in 1982. He receives the bachelor, master, and doctoral degree in electrical engineering from Institut Teknologi Bandung (ITB), Indonesia, in 2005, 2008, and 2013, respectively. He is currently a faculty member of the School of Electrical Engineering and Informatics, ITB. His research interests include medical signal \& image processing for ehealth applications, biomedical spectroscopy. Dr. Setiawan is a member of the IEEE, professional engineer (IPM), and ASEAN Engineer Registered. Email: awsetiawan@stei.itb.ac.id 